\title{
Emergency care in case of acute psychotic and/or manic symptoms: Lived experiences of patients and their families with the first interventions of a mobile crisis team. A phenomenological study
}

\author{
Daggenvoorde Thea, MSc PhD-candidate RN, Dimence Group, Deventer, The Netherlands
}

Gijsman Harm J., MD PhD Pro Persona Mental Health, Nijmegen, The Netherlands;

Goossens Peter J.J., Prof PhD APRN RN FEANS Dimence Group, Deventer, The Netherlands. Radboud university medical center, Radboud Institute for Health Sciences, IQ healthcare, Nijmegen, The Netherlands. GGZ-VS, Institute for Education of Clinical Nurse Specialist in Mental Health, Utrecht, The Netherlands. University Centre for Nursing and Midwifery, Department of Public Health, Faculty of Medicine and Health Sciences, Ghent University, Ghent, Belgium

Background: In case of acute mental health care problems, such as acute psychotic and/or manic symptoms, patients are typically referred to some sort of mobile mental health crisis team. These patients often are in a confused and disorganized state, what can hamper the communication. Guidelines for treatment recom- mend availability of mental health crisis services 24 hours a day and assessments at home. Nevertheless evidence based standard procedures are not available yet. A mental health crisis often has a great and long- lasting emotional impact for both patients and their families. Little is known about the lived experiences of these patients and their family members, during the first contact with the mobile crisisteam.

Objectives: In this study the lived experiences of patients with a psychotic or bipolar disorder and their fam-ilies during the first contact with a mobile crisis team were explored.

Methods: A qualitative, phenomenological study design was used. Open individual interviews with ten pa- tients and ten family members were held. Content data-analysis was conducted.

Results: A number of the patients missed communication about their condition and decisions being made. They had the feeling that they had no influence whatsoever on what the crisis team decided. Other patients, however, reported feeling understood and could easily accept the advice of the crisis team. Several patients reported not being able to remember much about the crisis. Most of the patients who were already in treat- ment had a personal crisis plan, but often they did not know if the crisis team had used the plan. Some

reported that using the plan was helpful for them, so they knew what to do. Patients often felt stigmatized when the mobile crisis team was called in to help and especially when the police had to get involved. In general, family members felt heard by the crisis team. Both patients and family members described calmness, empathy, and understanding on the part of the professional as essential.

Conclusions: Focus explicitly on communication with the patient, despite the acute condition, enhances the chance of cooperation. Taking time for contact with both patients and family members could contribute to less stigmatization and escalation. The use of a personal crisis plan is helpful. 